\title{
Unique structure and property of cyclodextrin and its utility in polymer synthesis
}

\author{
Jie Hu, Rong Huang, Shunsheng Cao, Yinqun Hua
}

*School of Materials Science and Engineering, Jiangsu University, Zhenjiang 212013, China; fax: 86-511-8791919; e-mail: hujiechem@hotmail.com.

(Received: 19 May, 2008; published: 28 December, 2008)

\begin{abstract}
Owing to their unique structure and property, natural cyclodextrins and their chemically modified derivatives can include many kinds of organic monomers to form guest/host complexes. The formation of these complexes results in significant changes of the solubility and activity of the guest molecules, but without chemical modification. It is found that the complexed hydrophobic monomers can be successfully polymerized directly in water without any organic solvent or surfactant, which induces a green way to polymer synthesis. It is found that this new cyclodextrin-mediated polymerization method differs strongly from classical methods. The present article reviewed the formation and the structure of the cyclodextrin/monomer inclusion complexes, and the influence of cyclodextrins on polymer synthesis. At last, the possible theory of the cyclodextrin-mediated reaction is discussed.
\end{abstract}

Keywords: cyclodextrins, polymer synthesis, polymerization, inclusion complexes

\section{Introduction}

Cyclodextrins (CDs) are a group of structurally related cyclic oligosaccharides consisting of 6,7 , and 8 units of $\alpha-1,4$-linked glucopyranose, and are named alpha $(\alpha)$, beta $(\beta)$, and gamma $(\mathrm{y})-C D$, respectively. Owing to the unique structure of $C D s$, their molecules are cone-shaped with a somewhat hydrophobic central cavity and hydrophilic outer surface. The depth of the cavities for the three CDs is the same, about $7.9 \AA$, while the cavity diameters of $\alpha-, \beta$-, and $\gamma-C D$ are different, about 5,6 , and $8 \AA$, respectively. The main structural data of the three natural CDs are shown in Table 1. CD molecules exhibit a kind of cavity with the hydrophobic interior 'lined' with hydrogen atoms and lone pairs of glycosidic oxygen atoms. On the other hand, primary and secondary hydroxyl groups situated at the narrow and wider bases, respectively, endow them with hydrophilic character, as shown in Figure 1. The hydrophobic nature of the CD cavities facilitates their ability to act as host clathrates for both polar and nonpolar guests and to form inclusion complexes. Though limited by the sizes of the host CD cavities and the cross sections of included guests, CDs can include many kinds of small molecules, as well as polymers, to form guest/host complexes [1-5]. The formation of these complexes results in significant changes of the solubility and activity of the guest molecules, but without chemical modification. It is believed that hydrogen bonds and hydrophobic interactions are responsible for it, sometimes electrostatic also involved [6]. So, natural CDs and their chemically modified derivatives, e.g. methylated or hydroxypropylated CD, have been the subject of numerous investigations, and have been applied in many fields, e.g. enzyme modelling, separation technology, food chemistry, cosmetic, catalyzer and pharmaceutical industries, etc $[7,8]$. It is generally believed that in these cases 
cyclodextrins can solubilize and stabilize active compounds, decrease their undesirable side effects, increase their bioavailability, mask unpleasant flavors or odors, or transform gases or liquids into solid powders. In the three natural CDs, $\beta$ $\mathrm{CD}$ is the most accessible, the lowest-priced and generally the most useful. However, its solubility in water is not satisfying because of the intramolecular strong hydrogen bond between $\mathrm{HO}-2$ and $\mathrm{O}-3$. So, some more water-soluble chemically modified $\beta$ CDs are extensively utilized, e.g. randomly methylated $\beta-C D$ and 2 -hydroxypropyl- $\beta$ CD.

Recently, inclusion complexes of guest monomers, i.e., styrene, methacrylates, methacylamides, pyrrole, and aniline, etc., with host natural CDs or chemically modified CDs and their polymerization have attracted considerable attention [9-12]. Ritter $\mathrm{H}$ first reviewed the utility of natural CDs and their modified compounds in polymer synthesis in 2002 [13]. In the following years, more monomeric guests were complexed with CDs, and the supermolecular structure of the monomer/CD complexes, the effect of CDs on the polymerization process and the microstructure of obtained polymers were further studied. In the present paper, based on the unique structure and property of cyclodextrin, we reviewed the utility of CDs in polymerization, especially the difference of reaction characteristics and structure of the obtained polymer from those of traditional polymerization.

Tab. 1. Main structural data of cyclodextrins.

\begin{tabular}{lccc}
\hline Property & $\alpha-C D$ & $\beta-C D$ & $\gamma$-CD \\
\hline Number of glucopyranose units & 6 & 7 & 8 \\
Molecular weight $(\mathrm{g} / \mathrm{mol})$ & 972 & 1135 & 1297 \\
Solubility in water at $25^{\circ} \mathrm{C}(\%, \mathrm{w} / \mathrm{v})$ & 14.5 & 1.85 & 23.2 \\
Outer diameter $(\AA)$ & 14.6 & 15.4 & 17.5 \\
Cavity diameter $(\AA)$ & $4.7-5.3$ & $6.0-6.5$ & $7.5-8.3$ \\
Height of torus $(\AA)$ & 7.9 & 7.9 & 7.9 \\
Cavity volume $(\AA)$ & 174 & 262 & 427 \\
& & & \\
\hline
\end{tabular}

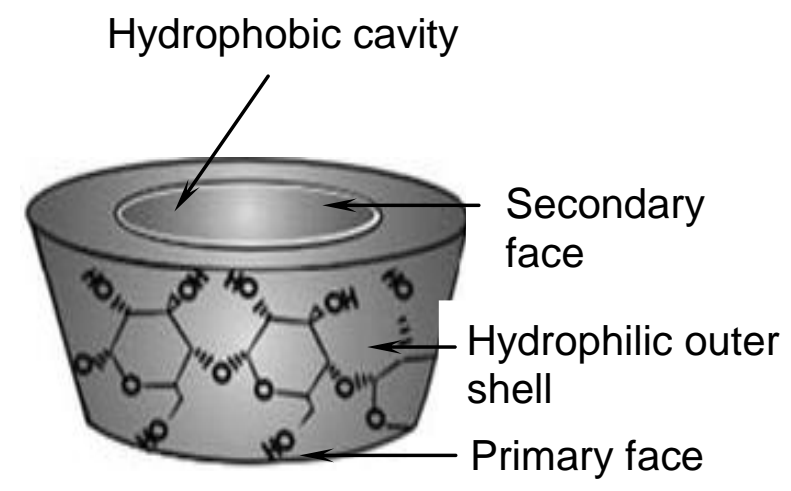

Fig. 1. Structure of cyclodextrins

\section{Cyclodextrin inclusion complexes formed with guest monomers}

Due to their relatively hydrophobic cavity and hydrophilic outer shell, CDs are able to form host-guest complexes with many organic monomers generally used in polymer 
preparation, e.g. styrene, methacrylates, methacrylamides, aniline, pyrrole, bithiophene, etc.. Usually, two different approaches can be followed to form monomer/CD inclusion compounds. The first is a "suspension" method in which preformed CD molecules containing only water of hydration are suspended into neat monomer to form the inclusion compound [9]. The second approach is a "precipitation" method, which is comparatively more convenient and thereby more commonly used. In this approach, a monomer is added directly to a CD aqueous solution and precipitation occurs as the guest molecules form the inclusion complexes with host CD molecules [14]. Nevertheless, it must be noticed that if more soluble modified CDs are used, such as randomly methylated $\beta-C D$, the inclusion compounds may dissolve in the aqueous to form a homogenous system.

It is believed that the formation of the inclusion complexes between cyclodextrins and monomers would markedly influence the physical and chemical properties of the monomers, especially their reactivity. So, it is important to make clear the characteristics of the inclusion complexes, e.g. the stoichiometry, inclusion coefficient, conformation of the monomer molecules in the cavity of CDs, etc.

The penetration of styrene and $\alpha$-methyl styrene in cavity of $\alpha-, \beta-$, and $\gamma-C D$ were studied by Yujuan Cao et al.[15, 16]. Results from quantum calculations show that both guest monomers penetrated into CDs' cavities deeply, and the complexes stability of each guest with different $C D$ is in the order $\alpha-C D>\beta-C D>\gamma-C D$. Furthermore, according to the TGA-DSC analysis and ${ }^{1} \mathrm{H}$ NMR titration, complex of $\alpha$-methyl styrene/ $\beta-C D$ is more stable than styrene/ $\beta-C D$, which may be result from the more interaction sites of $\alpha$-methyl styrene with $\beta-C D$ enhancing their van der Waals attraction. So, it is believed that the physical and chemical properties, especially reactivity of the two monomers would remarkably change.

Generally, styrene and acrylate monomers form inclusion complexes with $\beta-C D$ or its modified compounds in a 1:1 stoichiometry [17]. It is thought that acrylate monomers are incorporated into the $\beta-C D$ cavity with their reactive double bond first [18], just as shown in Figure 2. Theoretical investigations indicate that in styrene/ $\beta-C D$ inclusion complexes, the double bond of a styrene molecule also situates at the primary hydroxyl face, which is more stable than that at the second face [16].

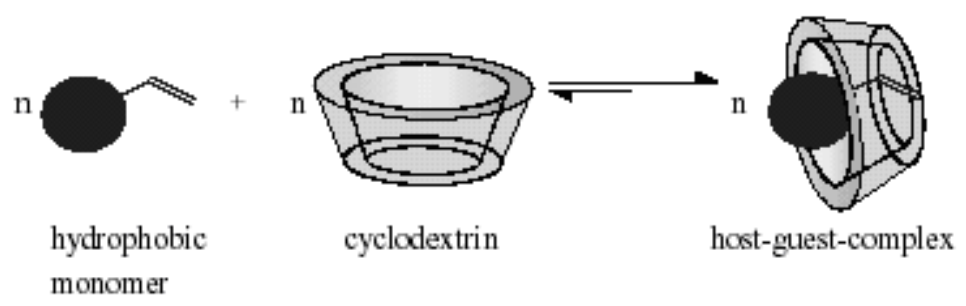

Fig. 2. Scheme of the incorporation of a monomer into the cavity of $\beta$-cyclodextrin.

Takashima $Y$ et al. [19] prepared a series of $\alpha-C D, \beta-C D$ and 2,6-di-O-dimethyl- $\beta$ $C D$ inclusion complexes with thiophenes in water and in crystalline states. The $\mathrm{X}$-ray analyses of $\alpha-C D /$ thiophene and $\beta-C D /$ bithiophene indicate 2:3 (CD/guest) inclusion complexes in the head to head channel structure. In contrast, the molecular structure of the 2,6-di-O-dimethyl- $\beta$-CD/bithiophene inclusion complex is formed to have a 1:1 stoichiometry with a cage type structure. Furthermore, the corresponding polymers of the three inclusion complexes can be obtained in water by $\mathrm{FeCl}_{3}$. 
In order to perform polymerization of styrene in a confined environment, the solid complex of guest styrene molecules included in the channels of host $\mathrm{Y}-\mathrm{CD}$ was prepared by Tamer Uyar et al. [1]. It is found that the experimental molar ratio of styrene to $\mathrm{Y}-\mathrm{CD}$ in the inclusion complex is $2: 1$, which is consistent with molecular modelling studies that indicate the $\mathrm{y}$-CD/two styrene molecular complex is the most energetically favorable. Once included in the CD cavities, the thermal stability of normally volatile bulk styrene to an elevated temperature is observed until the CD molecules themselves begin to degrade. In addition, the thermal degradation of host $\mathrm{Y}-\mathrm{CD}$ from the complex is observed to be different from that of pure $\mathrm{Y}-\mathrm{CD}$ due to codegradation of styrene and CD. Molecular modelling further suggest that the guest styrene molecules are arranged in a parallel and overlapped configuration at a separation distance of about $4.1 \AA$, the distance at which no $\pi-\pi$ interactions can be observed. Moreover, it is found [14] that there is a solid-phase transition from tetragonal to hexagonal packing of $\mathrm{Y}$-CD stacks upon dehydration of the styrene/CD inclusion complex crystal, and the solid state transformation between tetragonal and hexagonal channel crystal structures is surprisingly reversible upon the desorption or sorption of water.

Recently, ionic liquids become popular because of serious properties such as no vapor pressure and their potential as a solvent for polar substrates. It is interesting to investigate inclusion complexes of polymerizable ionic liquids, especially some insoluble ones, with CDs. Ritter $\mathrm{H}$ [20] pointed out that 1-butyl-3-vinylimidazolium bis(trifluoromethylsulfonyl)imide, a typical monomeric ionic liquid, could form 1:1 inclusion complex with $\beta-C D$, and only the anion was involved in the complex formation, which resulted in a spatial separation of the ion pair.

It is known that modelling the inclusion complexation of host CDs with organic guests can be a powerful tool, because it can potentially provide valuable and deep insights into the non-covalent, weak intermolecular interactions. Most computational studies of CDs focused on inclusion stoichiometry, conformation of guest monomers in the complexes, energies, preferred bonding orientations and so on. Owing to the relatively large molecular size of CDs, most of the modelling studies performed on CDs employed molecular mechanics, molecular dynamics, and Monte Carlo simulations [21,22]. Early quantum calculations on CDs were performed with semiempirical CNDO method in 1987 [23], thereafter, Yannakopoulou team [24] and Huang group $[25,26]$ carried out AM1 calculations on the inclusion complexes of $\beta$ CD. The PM3 method is also used to study the inclusion of different guest molecules into CDs, which proved to be a powerful tool for the conformation analysis of supermolecular systems [1, 27]. Furthermore, at higher level of quantum calculations, $\mathrm{ab}$ initio methods ${ }^{[28]}$ at the Hartree-Fork or the density functional theory levels with a minimal basis set were carried out. Calculations are useful for better understanding of such inclusion processes of CDs. The calculations can strengthen and supplement the conclusion from experiments.

\section{Effect of cyclodextrins on polymer Preparation}

\section{Effect of cyclodextrins on polymerization process}

In the 1970s, Ogata [29] and Maciejewski [30-32] reported the polymerization of inclusion complexes to prepare polyamides, poly(vinylidene chloride)s, and organosilicon oligomers. Monomers, like diamine and vinylidene chloride, are first 
complexed with CDs, and then inclusion polymerization of complexed monomers is carried out in aqueous or organic solvents.

Tab. 2. Characterization results for polymerization with different amount of $\beta-C D$.

\begin{tabular}{ccrccc}
\hline Reactions & Monomer & CD/g & Theoretical solids/\% & Coagulum/\% & $\mathrm{M}_{\mathrm{n}} \cdot 10^{3}$ \\
\hline 1 & DM & 0.0 & 22 & $>50 \%$ & 153 \\
2 & DM & 1.5 & 22 & 33 & 183 \\
3 & DM & 3.0 & 22 & 9 & 204 \\
4 & DM & 6.0 & 22 & 3 & 235
\end{tabular}

DM: dodecyl methacrylates

From the above mentioned, the water insoluble molecules may become water soluble simply by mixing with aqueous solutions of CDs, and it turned out that the complexed hydrophobic monomers can be successfully polymerized via free radicals in water. It has aroused great interest in many researchers. Octadecyl methacrylate and dodecyl methacrylate, highly hydrophobic monomers, are difficult to polymerize in aqueous media using traditional surfactant system, which is usually considered to be a consequence of the very low water solubility of the both monomers. In the presence of $C D$, these monomers can be successfully polymerized by emulsion polymerizations that do not involve the use of excessive levels of surfactant [33]. Just as shown in Table 2 [33], initially a conventional emulsion polymerization in the absence of CD was attempted, which produced over $50 \%$ of the charged monomer in the form of coagulum. On addition of CD, successful emulsion polymerizations were achieved, and average molecular weights were observed to increase as CD concentration increased. Similarly, using $\beta-C D$ as a complexing agent, the relative hydrophobic monomer $\mathrm{N}$-methacryloyl-11-aminoundecanoic acid can be polymerized radically in water without using any organic solvent or protective colloids [34]. Compared to the polymerization of the uncomplexed monomer in a homogeneous DMSO/water solution under similar conditions, the precipitation polymerization of the monomer/CD complex in water occurs much faster, and the resulting molecular weights are relatively higher. So, in presence of CDs, the influence of the hydrophilic character of the monomers on the polymerization is valuable to study the real role of CDs [35, 36]. It is found that the more hydrophobic the monomer, the higher the local concentration of monomer close to the active radical chain end of the polymer. This local concentration effect must lead to higher values of initial reaction rate.

The polymerization of cyclodextrin complexes of commercial monomers like styrene and (meth)acrylic derivatives thus opens an alternative route to polymer preparation. Phillip $\mathrm{H}$. Madison [37] researched the radical polymerization of tert-butyl methacrylate, cyclohexyl methacrylate, and 2-ethylhexyl methacrylate in the presence of methylated $\beta$-cyclodextrin. The resulting polymers exhibit numberaverage molecular weights as high as 140000 with PDl's as low as 3.2. Thus, hydrophobic, high molecular weight acrylic polymers are prepared in water with acceptable polydispersities and isolated yields as high as $86 \%$. More interesting, $80-$ $95 \%$ of the CD used in these reactions was recovered and ${ }^{1} \mathrm{H}$ NMR data and subsequent polymerization indicate that the carbohydrate can be recycled. Wenz et al. [38] synthesized the water-soluble nylon based on the solid-state polymerization of an inclusion compound between $\alpha, \omega$-amino acid and $\alpha-C D$. $\alpha-C D$ molecules 
threaded onto the nylon chains prevent the strong hydrogen bonds between the linear nylon chains, which makes great contribution to boost solubility.

Now, it is known that in presence of CDs, many hydrophobic monomers can be polymerized directly in water without any organic solvent or surfactant, which induces a green way to polymer synthesis with lower costs. Professor Helmut Ritter group is the most active in this field, and many organic monomers polymerized in water in presence of CDs were first reported by them [39-43]. Many observations have indicated that in presence of CDs, both the reaction rate and the monomer conversion are markedly elevated; some monomers even cannot polymerize in absence of CDs in similar conditions in water. Flosbach $C$ et al. [18] present an interesting way to synthesize powder clear coat resins by polymerization in aqueous solutions containing cyclodextrin. Upon polymerization, the resulting water-insoluble polymer cannot be complexed by CD due to sterical hindrance of the polymer chain. As a consequence, the free cyclodextrin remains in solution, whereas the resin separates from the aqueous solution. Compared to the common manufacturing of powder coat resins, no solvent is obtained as a byproduct, and the processes of distillation and crushing are also avoided.

Recently, the homo- and copolymerization of various methylated- $\beta C D$-complexed fluorinated monomers under surfactant-free conditions in aqueous media are also investigated [44-47]. Seen from Figure 3, after $120 \mathrm{~min}$, free monomer perfluorodecyl methacrylate and tert-butyl methacrylate is converted only about $70 \%$ and $50 \%$, respectively, much lower than those of the corresponding complexed monomers. It seems that the complexed monomers are more reactive in comparison to free ones. However, it must be pointed out that this comparison can only be qualitative because of the use of different solvents, initiator systems, and reaction temperatures. Most importantly, due to the fact that the reactions can be carried out in water without the use of any surfactants or cosolvents, this method of fluoropolymer production is environmental friendly and economical in comparison to traditional polymer synthesis, which might be of interest for industrial production of, e.g. coatings or emulgators.
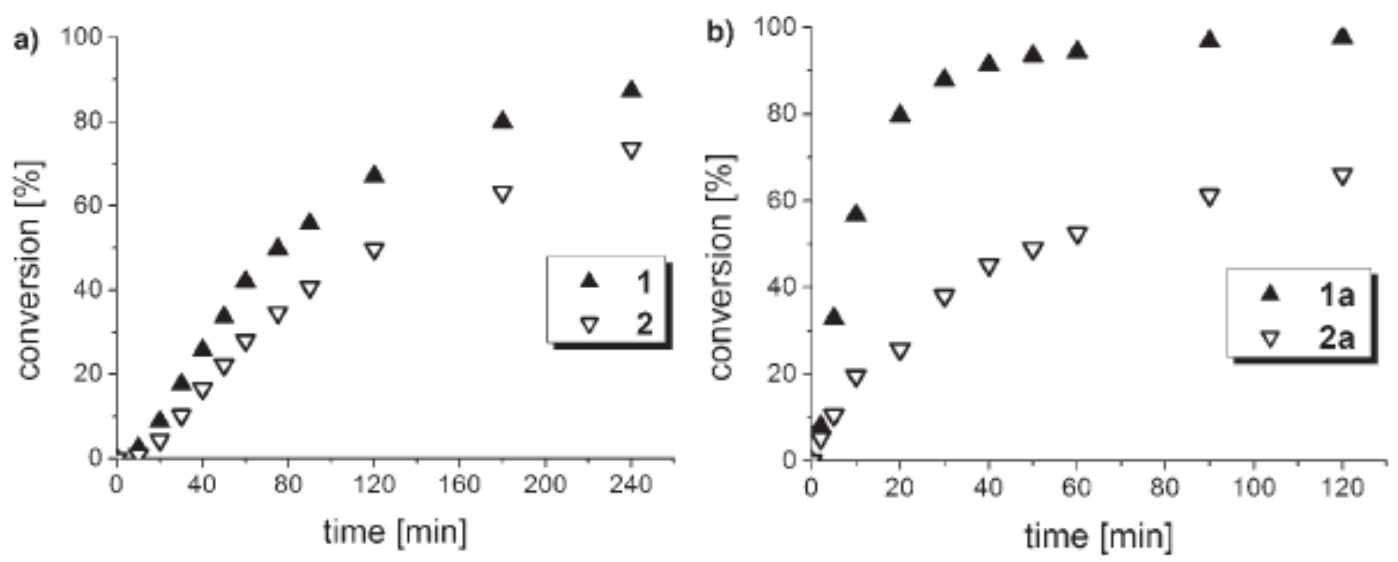

Fig. 3. Kinetic plots of the polymerization of perfluorodecyl methacrylate (1) and tertbutyl methacrylate (2) in benzene at $70{ }^{\circ} \mathrm{C}$ using AIBN as initiator (a), and of the corresponding methylated $\beta-C D$ complexes $1 \mathrm{a}$ and $2 \mathrm{a}$ in water at $25{ }^{\circ} \mathrm{C}$ using the redox initiator system $\mathrm{K}_{2} \mathrm{~S}_{2} \mathrm{O}_{8} / \mathrm{Na}_{2} \mathrm{~S}_{2} \mathrm{O}_{5}$ (b). 
Owing to the unique structure and property of CDs, some reactions, which hardly carry through by traditional methods, can favourably process in presence of CDs. For example, it is well established that dialkyl maleates such as diethyl maleate in general cannot give any polymers or oligomers. However, homopolymerization of CD-complexed diethyl maleate is observed, which shows cis/trans-isomerization during the polymerization [48]. It seems that the maleate radical is conversed to more stable fumarate before the propagation under the presence of cyclodextrin. For example, free radical polymerization of $\beta$-cyclodextrin complex of mesoionic 6 -oxo1,6-dihydropyrimidin-3-ium-4-olates in aqueous medium can lead to a photosensitive polymer [49]. In addition, a copolymer with methyl methacrylate prepared from the complex shows a different mass-dependent distribution in the incorporation in comparison to a copolymer prepared without CD in organic solvents. It is known that the copolymerization of water-soluble and water-insoluble monomers is difficult by classical emulsion polymerization because of the different solubilities of the monomers in water. For comparison, the copolymerization of hydrophobic monomer styrene with hydrophilic monomer $\mathrm{N}$-isopropyl acrylamide in the presence of $\mathrm{CD}$ and absence of CD are carried out under similar conditions [50]. It is found that, for all molar ratios of the incorporated monomer units, the weight averages of the copolymers obtained from complexed styrene and $\mathrm{N}$-isopropyl acrylamide are about 3 to 7 times higher than that of the corresponding copolymers obtained from uncomplexed styrene.

Another successful example of the cyclodextrin-mediated polymerization is the oxidative oligomerization of heterocyclic aromatic guests, such as pyrrole [12]. Due to the substantial m-electron delocalization along their backbones, these polymers show interesting (nonlinear) optical properties and become good electric conductors when oxidized or reduced. Additionally, the use of cyclodextrin to polymerize phenol derivatives in water are also investigated [51]. The enzyme catalyzed process is expected to be an alternative route for the preparation of phenol polymers without the use of toxic formaldehyde, which is a coupling comonomer for the synthesis of conventional phenolic resins. It is found that under similar condition only the presence of methylated $\beta-C D$ enables the polymerization of the phenol in water, while only dimmers or trimers could be obtained in absence of CD.

Recently, Osaki $\mathrm{M}$ et al. [52] reported new ring-opening polymerizations of lactones and lactides in presence of CDs, which can selectively give polyesters in high yields. They pointed out that the yields of the polyesters depend on the cavity size of CDs and on the structures of lactones, indicating that the reaction take place via inclusion of lactones in the CD cavity. The included monomer in the CD cavity is activated by the formation of hydrogen bonds between the hydroxyl group of CDs and the carbonyl oxygen of monomers in the initiation step. The products are found to be a polymer chain attached to the C-2-hydroxyl group of a single glucopyranose unit of $C D$ via an ester bond. The lactones are activated by the other remaining secondary hydroxyl groups to give the propagation step by way of insertions of monomers between CD and the polymer chain.

With crosslinking agent of various molar ratios of the host-guest complex consisting of styrene as the guest and methylated $\beta-C D$ as the host, Alupei I $C$ et al. described a new method to crosslink water soluble unsaturated polyester resins prepared from maleic anhydride and poly(ethylene glycol) in water [53]. Furthermore, successfully using the cyclopentadiene/methylated $\beta-C D$ inclusion complex in a Diels-Alder addition with the water soluble unsaturated polyester derived from poly(ethylene 
glycol) and maleic anhydride, they synthesized a new type of polyseudorotaxane [54]. Examination of the polypseudorotaxane and the model inclusion complex of the starting unsaturated polyester with the CD show that CD molecules are threaded onto the main chain in both cases.

Ding $Y$ et al. [55] prepared polyaniline salts by using solid polymerization in the presence of $\beta-C D$, which showed different physicochemical characteristics compared with that prepared in the absence of $\beta-C D$. When the molar ratio of aniline to $C D$ is $80 / 20$, the obtained polyaniline salt display higher crystallinity, conductivity and electrochemical properties, which are opposite to the condition that the molar ratio of aniline to $\beta-C D$ being $50 / 50$.

So, known from the above mentioned, due to the inclusion of the monomers into the CD cavities, this new cyclodextrin-mediated polymerization method differs strongly from classical methods. As an interesting observation, it is found that the reactivity ratios of the methylated $\beta-C D$ complexed monomers isobornyl acrylate (a) and $n-$ butyl acrylate $(b)\left(r_{a /} \beta-C D=0.3 \pm 0.1, r_{b} / \beta-C D=1.7 \pm 0.1\right)$ differ significantly from the values of the corresponding uncomplexed monomers in the mixture of $\mathrm{N}, \mathrm{N}$ dimethylformamide $(\mathrm{DMF}) / \mathrm{H}_{2} \mathrm{O}\left(r_{\mathrm{a}}=1.3 \pm 0.1, r_{b}=1.0 \pm 0.1\right)[56]$. Shown in Table 3 [57], further systematic investigations dealing with the influence of side groups of acrylates on the copolymerization behavior of various hydrophobic acrylate/CD complexes indicate that the interactions between monomer and cyclodextrin are liable substantially for the control of copolymerization reactivity ratios. It can be concluded that the better the guest monomer fits into the CD molecular cavities, the lower the reactivity ratio values.

Tab. 3. Reactivity ratios of methyl $\beta-C D$ complexed monomers $n$-butyl acrylate (1a), $\mathrm{n}$-hexyl acrylate (2a) and cyclohexyl acrylate (3a) in water and the corresponding uncomplexed monomers 1-3 in $\mathrm{DMF} / \mathrm{H}_{2} \mathrm{O}$ (9:1 vol.)

\begin{tabular}{cccc}
\hline \multicolumn{2}{l}{ monomer A monomer B } & $\mathrm{r}_{\mathrm{A}}$ & $\mathrm{r}_{\mathrm{B}}$ \\
\hline 1a (1) & $2 \mathrm{a}(2)$ & $1.01 \pm 0.01(1.01 \pm 0.01)$ & $1.04 \pm 0.01(0.91 \pm 0.03)$ \\
3a (3) & $2 \mathrm{a}(2)$ & $0.74 \pm 0.01(1.04 \pm 0.04)$ & $1.28 \pm 0.01(1.04 \pm 0.08)$ \\
$3 \mathrm{a}(3)$ & $1 \mathrm{a}(1)$ & $0.75 \pm 0.04(0.95 \pm 0.02)$ & $1.13 \pm 0.01(1.24 \pm 0.04)$ \\
\hline
\end{tabular}

Similarly, the chain-transfer constants may also markedly change in presence of CDs [58]. In the polymerizations of both uncomplexed monomers (methyl methacrylate (MMA) and styrene (St)) and monomers/CD complexes under similar conditions in the presence of chain-transfer agent $\mathrm{N}$-acetyl-L-cysteine, relatively high chain-transfer constants $(\mathrm{Cs})$ are found in the case of the complexed monomers in water (Cs $\mathrm{SMA}_{\mathrm{MM}}$ $\left.\mathrm{CD}=1.7 \pm 0.3, \quad \mathrm{Cs}_{\mathrm{St} / \beta-\mathrm{CD}}=2.6 \pm 0.3\right)$. In contrast, the chain-transfer constants in $\mathrm{DMF} / \mathrm{H}_{2} \mathrm{O}$ mixture are significantly lower (Cs MMA $=0.7 \pm 0.1$, and Cs $\mathrm{st}_{\mathrm{t}}=0.7 \pm 0.1$ ). This means that the molecular weight of polymers obtained from complexed monomers can be controlled more effectively than in the case of polymerization of the corresponding uncomplexed monomers.

Besides being used in general free radical polymerization, CDs are successfully applied in living radical polymerization, especially reversible addition fragmentation chain transfer polymerization (RAFT), to synthesize polymers with narrower polydispersity. Using 4,4'-di-(5-nonyl)-2,2'-bipyridyl as the ligand, which is complexed 
with large excess of methylated $\beta-C D$ in water (approximately 4-fold molar), the polymerization of methacrylate/methylated $\beta-C D$ complex is initiated by ethyl 2bromoisobutyrate in the presence of copper bromide as the catalyst. It is found that the polymerization has a living character, and the obtained polymer has a much lower polydispersity (PDI=1.3-1.8) [59, 60]. Zhang $\mathrm{F}$ et al. [61] believed that CD molecules act as both a solubilizer and a stabilizer, assisting the transportation of the water-insoluble RAFT agent into the polymerization loca and the stabilization of the low-molecular-weight radicals, which result in the decrease in polydispersity index.

\section{Effect of cyclodextrins on controlling structure of the polymer prepared}

As CDs more and more widely used in polymer synthesis, controlling the structure of the polymer prepared through the confined supermolecular microstructure of monomer/CD inclusion complexes has attracted a lot of attention. Tonelli A E et al. [9] pointed out that polymerization of monomers, like styrene, that were located as guests in the narrow channels of their cyclodextrin-inclusion compound crystals could potentially lead to polymers with microstructures that were distinct from those attained during homogeneous polymerizations of the same monomers, since certain microstructures resulting from homogeneous polymerizations might be prevented by the conformational restrictions placed on them by the narrow CD inclusion complex channels. For example, when propylene monomer is formed an inclusion complex with perhydrotriphenylene host and then free-radically polymerized in situ, the resulting polypropylene is found to be predominantly syndiotactic, consistent with the conformational modeling of polypropylene stereoisomers in channels appropriate to perhydrotriphenylene inclusion complexes [62]. Basing on the conformational modeling of stereoisomeric polystyrenes in narrow channels, they also suggested that polystyrene with unusual microstructures might be obtained via constrained polymerization of styrene in its $\mathrm{Y}$-CD inclusion complexes, where isotactic polystyrene should be obtained [9,63]. Similarly, modelling of acrylonitrile and syndiotactic- and isotactic-polyacrylonitrile/CD inclusion complexes using PM3 parameters show that the polymerization of acrylonitrile in CD channels produces predominantly isotactic polyacrylonitrile as a consequence of its improved fit compared with syndiotactic polyacrylonitrile [64]. However, further experimental polymerization of styrene inside the narrow channels of its $y-C D$ inclusion complex indicates that most $y-C D$ molecules slip off during polymerization and the channel structure is not preserved. Consequently, many guest styrene molecules polymerize outside of the host $\mathrm{Y}-\mathrm{CD}$ channels. Yet, a lightly rotaxanated structure has been obtained, where some threaded $\mathrm{y}$-CD molecules are permanently entrapped along the polystyrene chains after polymerization [65].

Recently, Zhu et al. [66] developed a method to control the polymeric architecture by introducing $\beta-C D$ into an $A_{2}+B B_{2}^{\prime}$ reaction system. Figure 4 indicates the proposed mechanism for the architecturally controlled polymerization. The polycondensation addition of divinylsulfone (an $A_{2}$ monomer) and 1-(2-aminoethyl)piperazine (a $\mathrm{BB}_{2}$ monomer) is a typical reaction for preparing hyperbranched polymers via the $A_{2}+$ BB' $_{2}$ strategy. Adding $\beta-C D$ into this system, inclusion complexation is induced by the similarities of the size and polarity between the 1-(2-aminoethyl)piperazine and the $\beta$ $C D$ cavity. Interestingly, one hydrogen atom of the primary amino group in the 1-(2aminoethyl)piperazine molecule is physically protected by the CD cavity, so the inclusion complex behaves as a bifunctional monomer during the polymerization. Therefore, the branching structure of the polymer chains can be easily controlled by 
merely adjusting the amount of $\beta$-CD. Furthermore, they extend this supramolecular synthetic approach into an $A_{2}+B_{4}$ system (divinylsulfone + hexamethylenediamine) [67]. By merely adjusting the amount of $\beta-C D$, a cross-linking gel, hyperbranched polymer, highly branched polymer, slightly branched polymer, or linear polymer can be obtained, respectively.

The synthesis of cyclic polymers in presence of $\beta-C D$ is another interesting example for controlling structure $[68,69]$. Generally, the basic synthetic approach of cyclic polymers is to form precursor polymers with two functional end groups and then to react them intramolecularly. However, it is difficult to obtain pure cyclic polymers. $\beta$ CD has 7 primary hydroxyl groups on one side of the ring and 14 secondary hydroxyl groups on the other side. As shown in Figure 5 [68], if vinyl groups are introduced into the secondary hydroxyl groups and the polymerization of vinyl groups is limited to the template monomer, both ends of the polymerized vinyl group will exist next to each other in the molecule. The closing of the ring in this monomer will be easier than for normal linear polymers. Because the number of vinyl groups in a $\beta-C D$ is controlled, the degree of polymerization of vinyl groups and its distribution will be narrow.

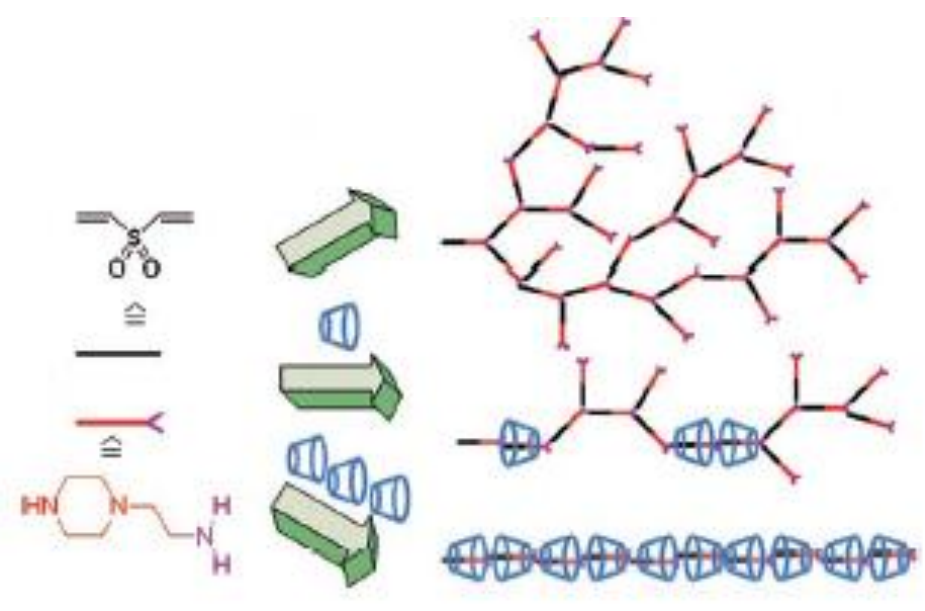

Fig. 4. Proposed mechanism for the cyclodextrin architecturally controlled polymerization.

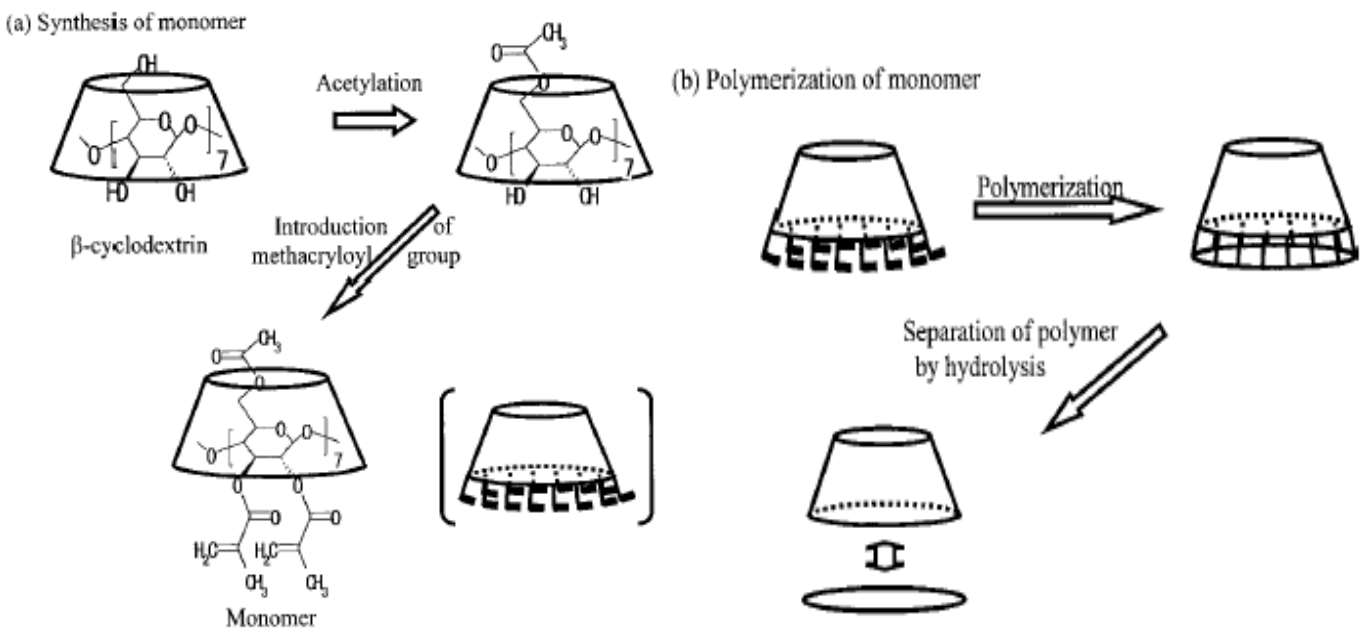

Fig. 5. Scheme of the synthesis of cyclic polymer with cyclodextrin. 


\section{Possible theory of the CD-mediated polymerization}

It is well known that the inclusion of monomers with CDs can markedly improve the solubility and stability of the monomers. The inclusion complexes can be successfully initiated in water to polymerize in a green way. Tonelli A E [9] pointed out that in order to obtain polymers, the CD molecules have to adopt continuous channel-type packing in which the monomer molecules reside in the channels and further react with each other once the polymerization is initiated (Figure 6(a)). They thought that the polymerization cannot be achieved in cage-type monomer/CD inclusion complexes, since the monomer molecules are entrapped in the CD cavities and are segregated from each other by neighboring host CD molecules (Figure 6(b)). However, Ritter $\mathrm{H}$ et al. $[13,58]$ believed, as shown in Figure 7, that during the freeradical polymerization of the complexed hydrophobic monomers, the CD ring would slip off step by step from the monomer during chain propagation and the polymer precipitates. The $C D$ remains finally in the aqueous phase due to its high water solubility. It is supported by the polymerization in presence of CDs, where the free radicals react quicker with butyl acrylate than with isobornyl acrylate [18]. This phenomenon is contributed to the fact that acrylate monomers are incorporated into the $C D$ cavities with their reactive double bond first. Consequently, the monomer molecules have to leave the complex with $C D$ to allow for a reaction at the reactive double bond. The weaker the interaction between monomer and CD is, the easier the monomer can leave the cyclodextrin's cavity. A small monomer like butyl acrylate is bound weaker to the cyclodextrin and can thus polymerize faster than isobornyl acrylate which fills the cavity well and can therefore exert stronger interactions with the host molecule. With this viewpoint in mind, it seems that the continuous channeltype packing of $C D$ molecules is not necessary for the polymerization of monomer/CD complexes. Later experiments performed by Tonelli A E et al. [65] themselves also indicate that most host CD molecules slip off during polymerization and the channel structure was not preserved.

So, from above mentioned, to achieve prolongation of the growing polymer chain, the $\mathrm{CD}$ have to slip off from the monomer. According to this mechanism, the complex stabilities and dynamics, which are influenced by the sterical demands of the monomers, must also have an influence on the overall rate of polymerization. According to kinetic studies on the free radical polymerization of CD-complexed styrene, it is indicated that the polymerization of the inclusion complex in water can be described by kinetics valid for the classical radical chain polymerization in solution [70]. The overall polymerization rate is proportional to the square root of initiator concentration, and the number average degree of polymerization is inversely proportional to the square root of initiator concentration. Furthermore, it is observed that the initial reaction rate increases with increasing of hydrophobic character of the complexed monomer [35]. This may be result from surface interactions between the complexed monomers and the phase separated growing polymer chain. It can be postulated that, the more hydrophobic the monomer, the higher the local concentration of monomer close to the active radical chain end of the polymer. This local concentration effect must lead to higher values of initial reaction rate. According to thermodynamic and kinetic considerations, $\mathrm{Li} \mathrm{S} \mathrm{J}$ et al. thought that increasing polymerization rate and monomer conversion may be result from the higher monomer reactivity and lower free energy ${ }^{[71]}$.

Building upon accepted current theories of free radical polymerization, Rimmer $\mathrm{S}$ et al. [72] pointed out that the concentration of the monomer in presence of CDs in 
aqueous phase will be higher than in the absence of CDs. This will have the effect of increasing the rate of initiation and thus the overall polymerization rate. As the molecular weight of the polymer increases, some CD molecules may be dethreaded and thus cause the growing polymer radical to precipitate.

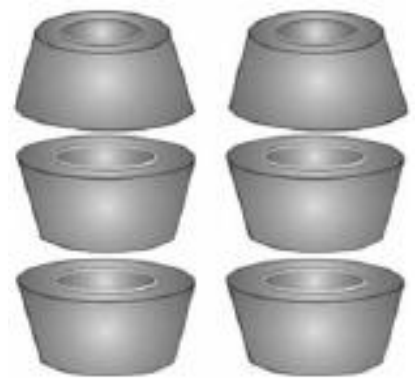

(a)

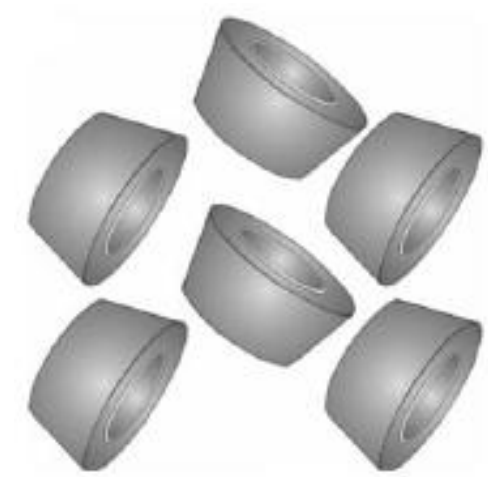

(b)

Fig. 6. (a) channel-type CD molecules; (b) cage-type CD molecules.

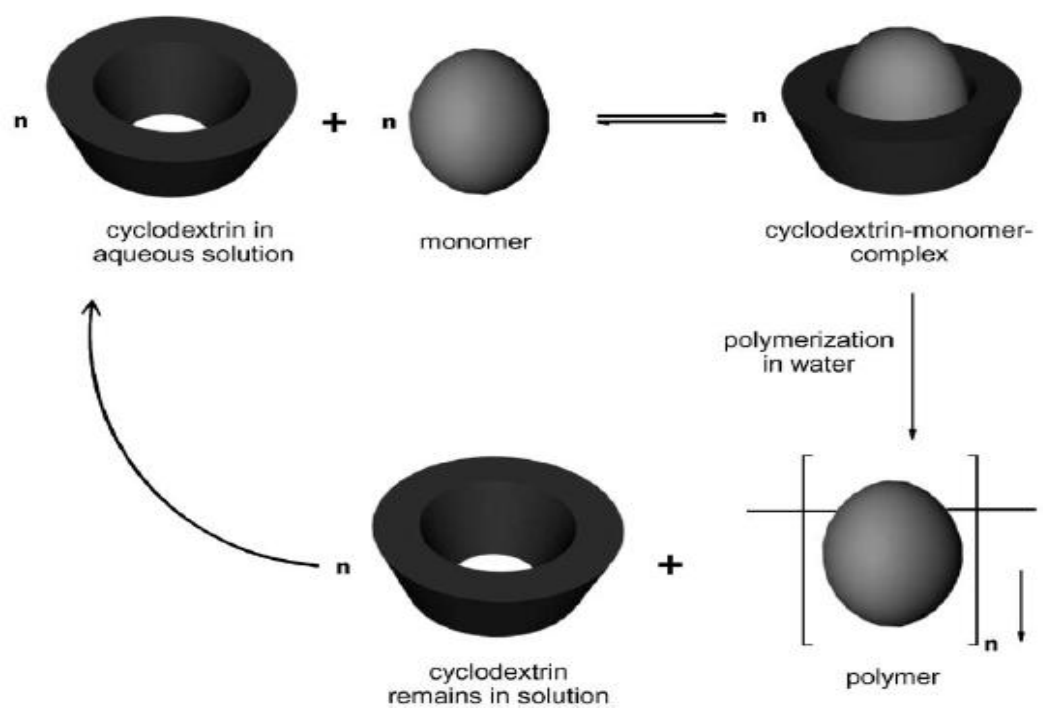

Fig. 7. Scheme of complexation and polymerization of cyclodextrin complexed monomers.

It is possible that this material will then enter a monomer droplet and may continue to propagate until termination occurs. Alternatively, precipitation may result in the formation of particles that act as new loci for polymerization. In this case, CD will have a further role in that the enhanced solubility of the monomer to these newly formed loci. In this scenario, if diffusion of monomer molecules is rate limiting, the rate of propagation would be increased. In both situations one would expect to observe an increase in molecular weight as a consequence of the reduced frequency of collisions between macroradicals. That is, in both cases, after precipitation of the growing polymer chain, termination can only occur when a second radical enters the polymerizing particle.

With the stabilizing mechanisms (entropic or electrostatic) in mind one would not expect a rigid non-charged cyclic compound such as CD to act as a colloid stabilizer. 
However, it is surprisingly discovered that the CD can stabilize some polymer colloids. It is also noticed that latices with lower CD concentrations display some settling over time, whereas those with higher $C D$ concentration remain well dispersed. This may be explained by the fact that a fraction of the CD molecules remains threaded on the polymer chain, then these macromolecules will have amphiphilic character. The polymer segments that are included will display hydrophilic behaviour while those segments that are not included will be hydrophobic in nature. These polymers may then be able to act as steric stabilizers. Experiments performed by Tonelli AE et al. [65] supported this viewpoint. It is found that not all CD molecules slip off. Yet, a lightly rotaxanated structure has been obtained, where some threaded CD molecules (about $15 \mathrm{wt} \%$ ) are permanently entrapped along the polymer chains after polymerization. Ritter $\mathrm{H}$ et al. [50] admitted that although the size exclusion chromatography diagrams suggested that there was no CD molecules contained in the polymer, the NMR spectra indicated that about $5 \%(\mathrm{w} / \mathrm{w})$ of CD molecules still remained in the polymers and cannot be removed by extraction.

\section{Conclusions}

From the investigations above mentioned, owing to their unique structure and property, natural CDs and their chemically modified derivatives can form inclusion complexes with many organic molecules. Generally, the reactive groups of the complexed guest molecules will keep their reactivity. So, hydrophobic monomers can be polymerized directly in water without any organic solvent or surfactant, which induces a green way to polymer synthesis with lower costs. This new cyclodextrinmediated polymerization method differs strongly from classical methods. Many observations have indicated that in presence of CDs, both the reaction rate and the monomer conversion are markedly elevated. And it is believed that during the freeradical polymerization of the complexed hydrophobic monomers, the CD ring would slip off step by step from the monomer during chain propagation and the polymer precipitates, so the CD can be circularly used. Furthermore, through the confined supermolecular microstructure of monomer/CD inclusion complexes, it is possible to control the structure of the polymer prepared.

Virtually, CDs have also been successfully applied in many other fields, such as food chemistry, cosmetic and pharmaceutical industries etc., although is not being described in detail here. Due to their special structure, we believe that CDs are still one of the hot points in the coming years.

\section{Acknowledgements}

Financial support of this study by National Natural Science Foundation of China (Approval No. 20704019) is gratefully acknowledged.

\section{References}

[1] Uyar, T.; El-Shafei, A.; Hacaloglu, J.; Tonelli, A.E. J Inclusion Phenom Macrocyclic Chem, 2006, 55,109.

[2] Uyar, T.; Rusa, C.C.; Hunt, M.A.; Aslan, E.; Hacaloglu, J.; Tonelli, A.E. Polymer, 2005, 46, 4762.

[3] Rusa, M.; Wang, X.W.; Tonelli, A.E. Macromolecules, 2004, 37, 18: 6898.

[4] Rusa, C.C.; Fox, J.; Tonelli, A.E. Macromolecules, 2003, 36, 2742.

[5] Mori, T.; Dong, T.; Yazawa, K. Macromol. Rapid Comm., 2007, 28, 2095. 
[6] Liu, Y.; Cao, R.; Chen, Y.; He, J.Y. J. Phys. Chem. B, 2008, 112, 1445.

[7] Karlson, L.; Thuresson, K.; Lindman B. Carbohydrate Polymers, 2002, 50, 219.

[8] Gregorio, G. Bioresource Technol., 2003, 90, 193.

[9] Uyar, T.; Rusa, M.; Tonelli, A.E. Macromol. Rapid Comm., 2004, 25(15), 1382.

[10] Wang, B.; He, J.; Sun, D.H.; Zhang, R.; Han, B.X.; Huang, Y. et al. Eur Polym J, 2005, 41 (10), 2483.

[11] Storsberg, J.; van Aert, H.; van Roost, C.; Ritter, H. Macromolecules, 2003, 36(1), 50.

[12] Storsberg, J.; Ritter, H.; Pielartzik, H.; Groenendaal, L. Adv. Mater., 2000, 12(8), 567.

[13] Ritter, H.; Tabatabai, M. Prog. Polym. Sci., 2002, 27, 1713.

[14] Uyar, T.; Hunt, M.A.; Gracz, H.S.; Tonelli, A.E. Cryst. Growth Des., 2006, 6 (5), 1113.

[15] Cao, Y.; Xiao, X.; Lua, R.; Guo, Q. J. Mol. Struct., 2003, 660, 73.

[16] Cao, Y.; Xiao, X.; Ji, S.; Runhua, L.; Guo, Q. Acta Part A, 2004, 60, 815.

[17] Caron, L.; Christine, C.; Tilloy, S.; Monflier, E.; Landy, D.; Fourmentin, S. et al. Supramol. Chem., 2002, 14, 11.

[18] Flosbach, C.; Herm, M.; Ritter, H.; Glockner, P. Prog. Org. Coat., 2003 , 48, 177.

[19] Takashima, Y.; Sakamoto, K.; Oizumi, Y.; Yamaguchi, H.; Kamitori, S.; Harada,

A. J. Incl. Phenom. Macro, 2006, 56, 45.

[20] Amajjahe, S.; Ritter, H. Macromolecules 2008, 41, 716.

[21] Liu, L.; Guo, Q-X. J. Phys. Chem. B., 1999, 103, 3461.

[22] Bonnet, P.; Jaime, C.; Morin-Alloy, L. J. Org. Chem., 2001, 66, 689.

[23] Kitagawa, M.; Hoshi, M.; Sakurai, H.; Inoue, Y.; Chujo, R. Carbohydr. Res., $1987,163,1$.

[24] Bosti, A.; Yannakopoulou, K.; Hadjoudis, E.; Waite, J. Carbohydr. Res., 1996, $283,1$.

[25] Huang, M.J.; Watts, J.D.; Bodor, N. Int. J. Quantum Chem., 1997, 64, 711.

[26] Huang, M.J.; Watts, J.D.; Bodor, N. Int. J. Quantum Chem., 1997, 65, 1135.

[27] Castro, R.; Berardi, M.J.; Cordova, E.; Deolza, M.O.; Kaifer, A.E.; Evanseck, J.D. J. Am. Chem. Soc., 1996,118, 10257.

[28] Lipkowitz, K.B. Chem. Rev., 1998, 98, 1829.

[29] Ogata, N.; Sanui, K.; Wada, J. J. Polym. Sci., Polym. Lett. Ed., 1976, 14, 459.

[30] Maciejewski, M.; Panasiewicz, M. J. Macromol. Sci., Chem. Part A, 1978, 12, 701.

[31] Maciejewski, M. J. Macromol. Sci. Chem. Part A, 1979, 13, 77.

[32] Maciejewski, M.; Gwizdowski, A.; Peczak, P.; Pietrzak, A. J. Macromol. Sci., Chem. Part A, 1979, 13, 87.

[33] Rimmer, S.; Tattersall, P. Polymer, 1999, 40, 5729.

[34] Jeromin, J.; Ritter, H. Macromolecules, 1999, 32, 5236.

[35] Bernhardt, S.; Glockner, P.; Theis, A.; Ritter, H. Macromolecules, 2001, 34, 1647.

[36] Steffens, C.; Choi, S.W.; Ritter, H. Macromol. Rapid Comm., 2006, 27, 7, 542.

[37] Phillip, H.M.; Timothy, E.L. Biomacromolecules, 2000, 1, 615.

[38] Steinbrunn, M.B.; Wenz, G. Angew. Chem., 1996, 108(18), 2274.

[39] Choi, S.W.; Ritter, H. Macromol. Rapid Comm., 2004, 25, 716.

[40] Steffens, C.; Kretschmann, O.; Ritter, H. Macromol. Rapid Comm., 2007, 28, 623.

[41] Sarvothaman, M.K.; Ritter, H. Macromol. Rapid Comm. 2004, 25, 1948.

[42] Choi, S.W.; Ritter, H. e-Polymers, 2007, no. 045. 
[43] Alupei, V.; Ritter, H. Macromol. Rapid Comm., 2001, 22, 1350.

[44] Choi, S.W.; Kretschmann, O.; Ritter, H.; Ragnoli, M.; Galli, G. Macromol. Chem. Phys., 2003, 204, 1475.

[45] Storsberg, J.; Ritter, H. Macromol. Chem. Phys., 2002, 203, 812.

[46] Cinar, H.; Kretschmann, O.; Ritter, H. Macromolecules 2005, 38, 5078.

[47] Kretschmann, O.; Ritter, H. Macromol. Chem. Phys. 2006, 207, 987.

[48] Choi, S.W.; Frank, W.; Ritter, H. React. Funct. Polym., 2006, 66, 149.

[49] Theis, A.; Ritter, H. Macromol. Chem. Phys., 2003, 204(10), 1297.

[50] Casper, P.; Glockner, P.; Ritter, H. Macromolecules, 2000, 33, 4361.

[51] Reihmann, M.H.; Ritter, H. Macromol Chem Phys, 2000, 201, 798.

[52] Osaki, M.; Takashima, Y.; Yamaguchi, H.; Harada, A. Kobunshi Ronbunshu, 2007, 64, 607.

[53] Alupei, I.C.; Alupei, V.; Ritter, H. Macromol. Rapid Comm., 2003, 24, 527.

[54] Alupei, V.; Alupei, I.C.; Ritter, H. Macromol. Rapid Comm., 2005, 26, 40.

[55] Ding, Y.; Abdiryim, T.; An, S.; Nurulla, I. J. Appl. Polym. Sci., 2008, 107, 3864.

[56] Glockner, P.; Ritter, H. Macromol. Rapid Comm., 1999, 20, 602.

[57] Bernhardt, S.; Glöckner, P.; Ritter, H. Polym. Bull., 2001, 46, 153.

[58] Glockner, P.; Metz, N.; Ritter, H. Macromolecules, 2000, 33, 4288.

[59] Kollisch, H.; Barner-Kowollik, C.; Ritter, H. Macromol. Rapid Comm., 2006, 27, 848.

[60] Storsberg, J.; Hartenstein, M.; Muller, A.H.E.; Ritter, H. Macromol. Rapid Comm., 2000, 21, 1342.

[61] Zhang, F.; Ni, P.H.; Xiong, Q.F.; Yu, Z.Q. J. Polym. Sci. Part A, 2005, 43, 2931.

[62] Tonelli, A.E. Macromolecules, 1991, 24, 3069.

[63] Hunt, M.A.; Jung, D.W.; Shamsheer, M.; Uyar, T.; Tonelli, A.E. Polymer, 2004, $45,1345$.

[64] Yang, H.; El-Shafei, A.; Schilling, F.C.; Tonelli, A.E. Macromol. Rapid Comm., 2007, 16, 797 .

[65] Uyar, T.; Gracz, H.S.; Rusa, M.; Shin, I.D.; El-Shafei, A.; Tonelli, A.E. Polymer, 2006, 47, 6948.

[66] Chen, L.; Zhu, X.; Yan, D.Y.; Chen, Y.; Chen, Q.; Yao, Y.F. Angew. Chem. Int. Ed., 2006, 45, 87.

[67] Wan, H.S.; Chen, Y.; Chen, L.; Zhu, X.Y.; Yan, D.Y.; Li, B. et al. Macromolecules, 2008, 41, 465.

[68] Saito, R.; Okuno, Y.; Losayashi, H. J. polym. Sci. Part A, 2001, 39, 3539.

[69] Saito, R.; Kobayashi, H. Macromolecules, 2002, 35, 7207.

[70] Ritter, H.; Steffens, C.; Storsberg, J. e-polymers, 2005, 034.

[71] Li, S.J.; Hu, J.; Liu, B.L.; Li, H.; Wang, D.Q.; Liao, X.F. Polymer, 2004, 45, 1511.

[72] Rimmer, S.; Tattersall, P.I. Polymer, 1999, 40, 6673. 